\title{
Suco de uva suplementado com Lactobacillus acidophilus e oligofrutose
}

\author{
Grape juice suplemented with \\ Lactobacillus acidophilus and oligofrutose
}

\author{
Joice Sifuentes dos Santos ${ }^{1}$; Ana Augusta Odorissi Xavier²; \\ Priscila Boneventi ${ }^{3}$; Roberta B. de Souza ${ }^{4}$; Sandra Garcia ${ }^{5 *}$
}

\section{Resumo}

\begin{abstract}
Alimentos simbióticos contendo probióticos e prebióticos são benéficos para a saúde humana. $\mathrm{O}$ objetivo deste trabalho foi desenvolver um suco de uva simbiótico, como alternativa a bebidas lácteas probióticas, contendo o probiótico Lactobacillus acidophilus e o prebiótico oligofrutose. Para avaliar a melhor formulação do suco, foi realizado um planejamento fatorial $2^{2}$. O suco comercial pasteurizado foi inoculado com 10 e $15 \%$ de oligofrutose e $10^{7}$ e $10^{8} \mathrm{UFC} / \mathrm{mL}$ de L. acidophilus, mantido sob refrigeração por 8 dias. $\mathrm{O}$ pH dos sucos elaborados $(2,86$ - 3,52) aumentou em relação ao controle $(2,69)$. Observou-se que a formulação contendo $10 \%$ de oligofrutose e $10^{8} \mathrm{UFC} / \mathrm{mL}$ de L. acidophilus foi a que apresentou contagem de bactérias lácticas $\left(1,25 \times 10^{7} \mathrm{UFC} / \mathrm{mL}\right)$ dentro da legislação para utilização da alegação de produto probiótico. Assim, esta formulação foi escolhida para realizar a análise sensorial. O teste triangular demonstrou que houve diferença significativa entre o suco simbiótico e o suco controle $(\mathrm{p} \leq 0,001)$. Por outro lado, no teste afetivo de preferência (escala hedônica de 9 pontos), as notas atribuídas pelos 18 provadores ao suco controle $(6,67 \pm 1,27)$ e ao simbiótico $(5,56 \pm 2,56)$ não diferiram estatisticamente $(\mathrm{p}>0,05)$.
\end{abstract}

Palavras-chave:

\begin{abstract}
Synbiotic foods containing probiotics and prebiotics are good for human health. The aim of this work was to develop a symbiotic grape juice, as alternative to dairy probiotic products, containing the probiotic culture Lactobacillus acidophilus and oligofrutose as prebiotic. To evaluate the best juice formulation, was realized a $2^{2}$ factorial planning. The juice was kept under refrigeration for 8 days. The symbiotic juice $\mathrm{pH}(2.86-3.52)$ increased in comparison to the control (2.69). It was observed that the formulation containing $10 \%$ oligofrutose and $10^{8} \mathrm{CFU} / \mathrm{mL}$ of $L$. acidophilus presented the lactic acid bacterial counting $\left(1.25 \times 10^{7} \mathrm{CFU} / \mathrm{mL}\right)$ according to the legislation for probiotic claim. This formulation was
\end{abstract}

\footnotetext{
1 Farmacêutica e Bioquímica - Tecnóloga de Alimentos, Doutoranda do Programa de Pós-Graduação em Ciência de Alimentos da Universidade Estadual de Londrina, Bolsista CNPq do Brasil.

2 Farmacêutica e Bioquímica - Tecnóloga de Alimentos, Mestre em Ciência de Alimentos da Universidade Estadual de Londrina, Bolsista CNPq do Brasil.

3 Bióloga, Mestre em Ciência de Alimentos da Universidade Estadual de Londrina, Bolsista CAPES.

4 Bióloga, Mestre em Ciência de Alimentos da Universidade Estadual de Londrina, Bolsista CNPq do Brasil.

5 Engenheira de Alimentos, Doutora em Ciência de Alimentos, Professora do Departamento de Ciência e Tecnologia de Alimentos da Universidade Estadual de Londrina. Telefone: (43) 3371-4565.

* Autor para correspondência
} 
selected for sensorial evaluation. The triangular test showed a significant difference between synbiotic and control juice $(\mathrm{p} \leq 0.001)$. In the other hand, in the affective test of preference (hedonic scale of 9 points), the scores given by the 18 painelists to the control $(6.67 \pm 1.27)$ and symbiotic $(5.56 \pm 2.56)$ juices were not statistically different $(\mathrm{p}>0.05)$.

Key words:

\section{Introdução}

Probióticos são definidos como suplementos microbianos que influenciam positivamente o organismo e aumentam de maneira significativa o valor nutritivo e terapêutico dos alimentos, através do equilíbrio microbiano intestinal e das funções fisiológicas do trato intestinal humano. Da mesma forma, alimentos probióticos são definidos como alimentos contendo microrganismos, que possuem efeito benéfico sobre a microbiota intestinal e as funções fisiológicas do trato intestinal humano. $\mathrm{Na}$ sua grande maioria, os produtos probióticos do mercado são de origem láctea, e a disponibilidade de produtos não lácteos ainda é reduzida.

Dentre os diversos gêneros que integram este grupo, destacam-se Bifidobacterium e Lactobacillus e, em particular, a espécie Lactobacillus acidophilus. Além dos benefícios em termos de nutrição e de saúde que proporcionam, as culturas probióticas podem também contribuir para melhorar o sabor do produto final, possuindo a vantagem de promover acidificação reduzida durante a armazenagem pósprocessamento (GOMES; MALCATA, 1999). As culturas utilizadas como probióticas devem tolerar o processamento e manter a viabilidade celular durante o armazenamento. A sobrevivência das células no produto irá depender de diversos fatores, como $\mathrm{pH}$ e presença de preservantes (CHARTERIS et al., 1998).

Os frutooligossacarídeos (FOS) consistem de moléculas de sacarose, nas quais uma ou duas outras unidades de frutose são adicionadas por ligações $\beta-1,2$ à molécula de frutose original. $O$ grau de polimerização varia de 2 a 10 unidades. Os derivados são encontrados naturalmente em vegetais e plantas como alcachofra, raiz de chicória, dália, dente de leão, alho, cebola, banana e outras. No entanto, a quantidade encontrada nesses alimentos é pequena, exigindo consumo elevado para se obter o efeito funcional esperado. A oligofrutose (inulina hidrolisada enzimaticamente) é considerada um prebiótico. Prebiótico é um ingrediente alimentar que não é hidrolisado por enzimas digestivas humanas no trato gastrintestinal superior e afeta beneficamente o indivíduo, por estimulação seletiva do crescimento e/ou atividade de bactérias no cólon (GIBSON; ROBERFROID, 1995). Alimentos probióticos são definidos como aqueles que contêm culturas de microrganismos que afetam beneficamente a saúde do consumidor por melhorar seu balanço microbiano intestinal (FULLER, 1989). Quando prebióticos e probióticos estão presentes simultaneamente, o termo utilizado é simbiótico (JAIN et al., 2004).

Entre as propriedades prebióticas estudadas, existe um consenso de que os FOS modificam o habitat intestinal, causando aumento no bolo fecal, normalização da freqüência fecal, efeito prebiótico (aumenta o número de bactérias e/ou atividade do número de bifidobactérias e bactérias lácticas, no intestino humano), estímulo da absorção de cálcio dos alimentos, modulação do metabolismo lipídico e prevenção de casos de câncer (GERMAN et al., 1999; RAO, 2001).

Desta forma, o objetivo do presente trabalho foi avaliar a sobrevivência de Lactobacillus acidophillus em suco de uva com adição de oligofrutose e verificar sua aceitação sensorial.

\section{Materiais e Métodos}

\section{Materiais}

Foi utilizado suco de uva pasteurizado, não fermentado, não alcoólico, reconstituído, sem conservantes, com validade de 1 ano. Cada $200 \mathrm{~mL}$ 
do suco (segundo o fabricante) era composto por $30 \mathrm{~g}$ de carboidratos e $0,3 \mathrm{mg}$ de ferro. O produto não continha proteína, gordura ou sódio, e teor não significativo de cálcio.

A cultura de Lactobacillus acidophilus La-5 da empresa Christian Hansen, liofilizada e de uso direto, foi dissolvida assepticamente em suco de uva. O suco, após inoculação e homogeneização, foi armazenado por um período de 8 dias sob refrigeração a $8{ }^{\circ} \mathrm{C}$. Ao mesmo tempo em que a cultura foi adicionada ao suco, também foi realizada uma contagem de bactérias lácticas para avaliação.

A oligofrutose utilizada foi da marca Orafti, comercializada sob o nome Raftilose ${ }$.

\section{Métodos}

Foram realizadas análises de $\mathrm{pH}$ e contagem de Lactobacillus acidophilus no tempo zero (logo após adição de L. acidophilus e oligofrutose) e 2, 4 e 8 dias após a adição. As análises de $\mathrm{pH}$ e contagens foram feitas em duplicata.

$\mathrm{O} \mathrm{pH}$ das amostras foi determinado utilizando pHmetro Tecnal.

Para avaliar o crescimento de Lactobacillus acidophilus, foram adicionados $10 \mathrm{~mL}$ de suco a $90 \mathrm{~mL}$ de água peptonada. A seguir, procedeu-se a diluições em série e inoculou-se $1 \mathrm{~mL}$ em placas de Petri estéreis, adicionando em seguida 15-20 mL de meio MRS (ágar de Man, Rogosa \& Sharpe). As placas foram incubadas a $37^{\circ} \mathrm{C}$ por $36 \mathrm{~h}$.

A contagem de microrganismos indicadores (coliformes a $35^{\circ} \mathrm{C}$ ) foi realizada através do método do NMP (número mais provável). O consumo das amostras não apresentou risco de contaminação de origem fecal aos provadores, uma vez que havia ausência deste tipo de microrganismos. Foram adicionados $10 \mathrm{~mL}$ de suco $\left(10^{-1}\right)$ a $90 \mathrm{~mL}$ de água peptonada e procedeu-se mais 2 diluições $\left(10^{-2} \mathrm{e}\right.$ $10^{-3}$ ). Foi inoculado $1 \mathrm{~mL}$ de cada dilução em tubo contendo caldo VB (verde brilhante) e tubo de Durhan invertido. Os tubos foram incubados a 35 ${ }^{\circ} \mathrm{C} / 24-48$ horas.

A possível interação entre o teor de oligofrutose e a contagem de BAL no tempo zero (variáveis independentes) sobre o crescimento de BAL ao término de 8 dias de incubação (variável dependente) foi avaliada através da técnica de superfície de resposta. Para isso, foi utilizado um planejamento experimental fatorial $2^{2}$. Os níveis encontram-se na Tabela 1. Para verificar se o tratamento realizado promoveu diferença no sabor do suco adicionado de sistemasimbiótico, procederam-seanálisessensoriais no produto utilizando um painel composto por 18 provadores não-treinados recrutados dentre alunos, professores e funcionários da Universidade Estadual de Londrina. Foram realizados os testes triangular, onde se apresenta ao provador três amostras, sendo duas iguais entre si e uma diferente, e verifica-se a capacidade do provador de reconhecer qual amostra é a diferente, e o teste afetivo de preferência mediante escala hedônica de 9 pontos ( 1 - desgostei muitíssimo; 9 - gostei muitíssimo). Os testes foram praticados em cabines individuais do laboratório de análise sensorial, durante o período vespertino, com a luz vermelha acesa para mascarar a aparência das amostras, requisitando-se aos provadores o enxágüe da boca com água potável a temperatura ambiente, antes de provar a amostra. Cada amostra foi fornecida em copos descartáveis brancos contendo $30 \mathrm{~mL}$ de suco, e foram apresentadas aos provadores em ordem aleatória. 
Tabela 1. Planejamento experimental contendo as variáveis e os níveis usados nos experimentos.

\begin{tabular}{ccc}
\hline Amostra & $\begin{array}{c}\text { L. acidophilus } \\
(\mathrm{UFC} / \mathrm{mL})\end{array}$ & $\begin{array}{c}\text { Oligofrutose } \\
(\mathrm{g} / 100 \mathrm{~mL})\end{array}$ \\
\hline Controle & 0 & 0 \\
1 & $10^{7}$ & 10 \\
2 & $10^{7}$ & 15 \\
3 & $10^{8}$ & 10 \\
4 & $10^{8}$ & 15 \\
\hline
\end{tabular}

As análises estatísticas foram realizadas utilizando-se o programa Statistica 6.0, submetendose os resultados à análise de variância (ANOVA).

\section{Resultados e Discussão}

Os resultados obtidos a partir de análises microbiológicas e de $\mathrm{pH}$ dos sucos de uva adicionado de Lactobacillus acidophilus e oligofrutose, ao longo de 8 dias de incubação, podem ser observados nas Figuras 1 e 2.

Todas as amostras apresentaram o mesmo comportamento de $\mathrm{pH}$ durante o experimento. $\mathrm{O}$ valor de $\mathrm{pH}$ permaneceu constante até o $4^{\circ}$ dia de incubação, sofrendo pequena diminuição nos dias posteriores (Figura 1). Estes resultados assemelhamse aos obtidos por Martínez-Villaluenga et al. (2006), que analisaram a influência da adição de oligossacarídeos na sobrevivência de probióticos em leite fermentado refrigerado.

A amostra controle (sem adição de $L$. acidophilus e oligofrutose) não continha bactérias lácticas naturalmente presentes (Figura 2). Nas amostras 1 e 2 , onde foi adicionado $10^{7} \mathrm{UFC} / \mathrm{mL}$ de L. acidophilus, não houve sobrevivência das bactérias lácticas até o fim dos 8 dias de incubação, tanto com adição de 10 ou $15 \%$ de oligofrutose. Pode-se observar que a maior sobrevivência de microrganismos ocorreu na amostra 3, onde adicionou-se $10^{8}$ UFC de L. acidophilus $/ \mathrm{mL}$ e $10 \%$ de oligofrutose, sendo esta a formulação escolhida para realizar a análise sensorial. Esta formulação atingiu o valor estabelecido pela legislação para contagem de bactérias probióticas, que é de $10^{7}$ UFC/mL (BRASIL, 2000). As contagens realizadas evidenciaram que na amostra 3 houve manutenção do número de microrganismos até o oitavo dia, o mesmo não ocorreu com a amostra 4 , onde foi inoculada a mesma quantidade de microrganismos, porém quantidade superior de oligofrutose.

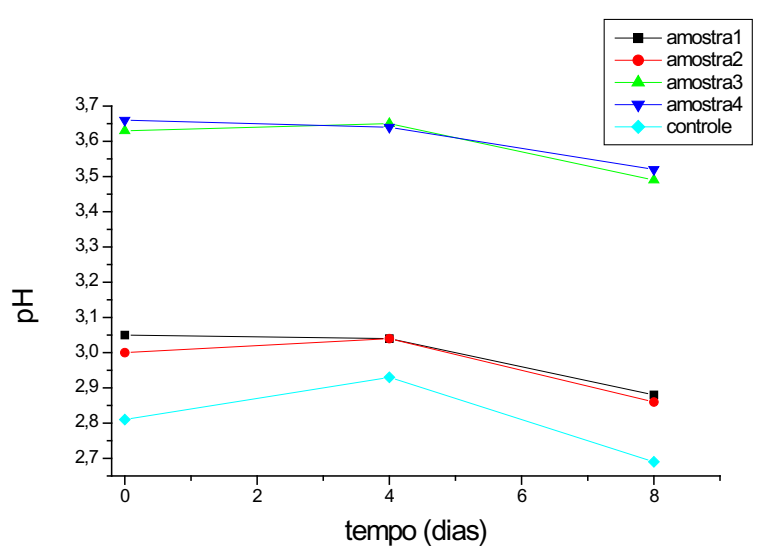

Figura 1. Valores de $\mathrm{pH}$ das amostras de suco de uva simbiótico ao longo de 8 dias de incubação. Amostra $1=10^{7} \mathrm{UFC} / \mathrm{mL}$ de L. acidophilus (La-5) e $10 \%$ de oligofrutose; amostra $2=10^{7} \mathrm{UFC} / \mathrm{mL}$ de La-5 e $15 \%$ de oligofrutose; amostra $3=10^{8} \mathrm{UFC} / \mathrm{mL}$ de La-5 e $10 \%$ de oligofrutose; amostra $4=10^{8} \mathrm{UFC} / \mathrm{mL}$ de La-5 e $15 \%$ de oligofrutose; controle $=$ suco sem adição de La-5 e oligofrutose. 


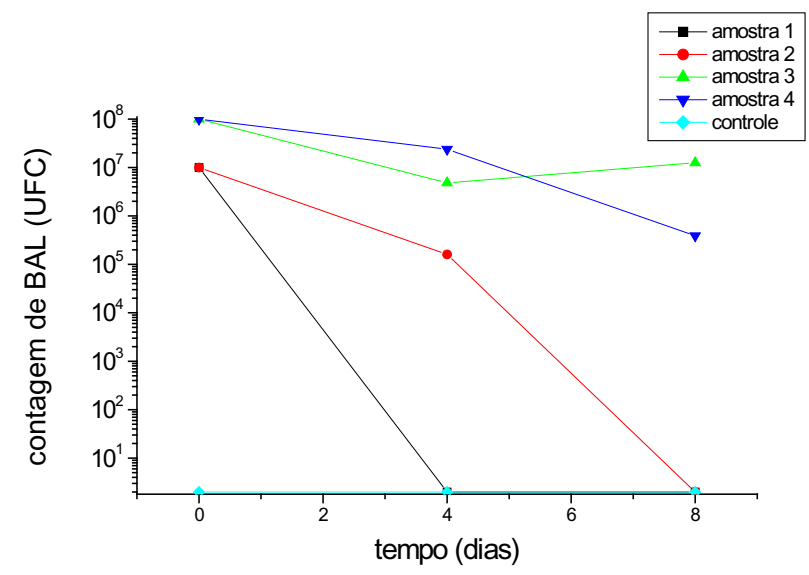

Figura 2. Contagem de bactérias lácticas (BAL) em suco de uva simbiótico ao longo de 8 dias de incubação Amostra $1=10^{7} \mathrm{UFC} / \mathrm{mL}$ de L. acidophilus (La-5) e 10\% de oligofrutose; amostra $2=10^{7} \mathrm{UFC} / \mathrm{mL}$ de La-5 e $15 \%$ de oligofrutose; amostra $3=10^{8} \mathrm{UFC} / \mathrm{mL}$ de La-5 e $10 \%$ de oligofrutose; amostra $4=10^{8} \mathrm{UFC} / \mathrm{mL}$ de La-5 e $15 \%$ de oligofrutose; controle $=$ suco sem adição de La-5 e oligofrutose.

Na Figura 3 observa-se a superfície de resposta do crescimento de bactérias láticas sob a influência da quantidade de oligofrutose e concentração inicial de bactérias lácticas. Não houve interação entre as variáveis quantidade de oligofrutose e concentração inicial de inóculo, não exercendo efeito sobre a contagem de BAL. A concentração inicial de inóculo influenciou a contagem de BAL, embora em pequena proporção.

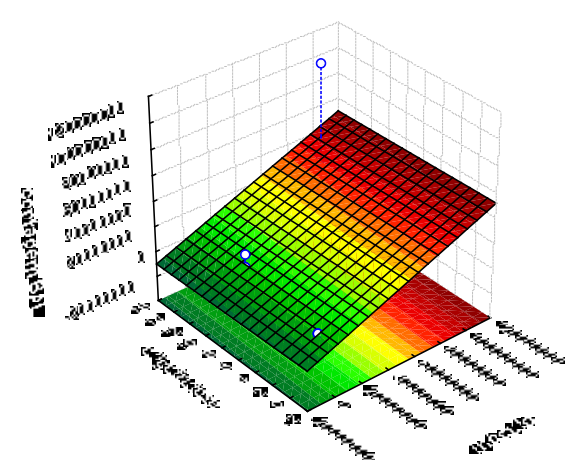

$4 \times 10^{7} \mathrm{UFC} / \mathrm{mL}$ $2 \times 10^{7} \mathrm{UFC} / \mathrm{mL}$

Figura 3. Superfície de resposta do crescimento de bactérias lácticas (BAL) em $\mathrm{UFC} / \mathrm{mL}$, utilizando 2 fatores ( $\%$ de oligofrutose e contagem de BAL no tempo zero) em 2 níveis.
Quanto ao crescimento de coliformes a $35{ }^{\circ} \mathrm{C}$, observou-se ausência em todas as amostras.

A avaliação sensorial, através do teste triangular, demonstrou que houve diferença significativa entre o suco adicionado de sistema simbiótico e o suco controle, com $\mathrm{p} \leq 0,001$. Apenas 2 provadores não detectaram diferença entre as amostras. Por outro lado, no teste afetivo de preferência, as notas atribuídas pelos 18 provadores ao suco controle e ao simbiótico não diferiram estatisticamente $(\mathrm{p}>0,05)$. As notas atribuídas aos sucos são apresentadas na Figura 4. O suco controle obteve nota média $6,67 \pm 1,27$ e o suco simbiótico 5,56 $\pm 2,56$, utilizando uma escala hedônica de 9 pontos. Para notas abaixo de 5 considera-se o produto rejeitado, nota igual a 5 indiferença, e notas superiores a 5 significam aceitação do produto. Pode-se observar que o suco controle foi rejeitado por apenas 1 pessoa, enquanto que o suco simbiótico por 7 pessoas. Nenhum dos provadores demonstrou indiferença em relação a ambos. O suco simbiótico recebeu uma nota máxima. Diversos provadores relataram gosto mais adocicado do suco adicionado de sistema simbiótico, o que pode estar relacionado ao gosto levemente doce apresentado pela oligofrutose. Para solucionar este problema, poder-se-ía reduzir ou, se necessário, retirar da formulação o edulcorante utilizado, aproveitando o poder adoçante da oligofrutose.

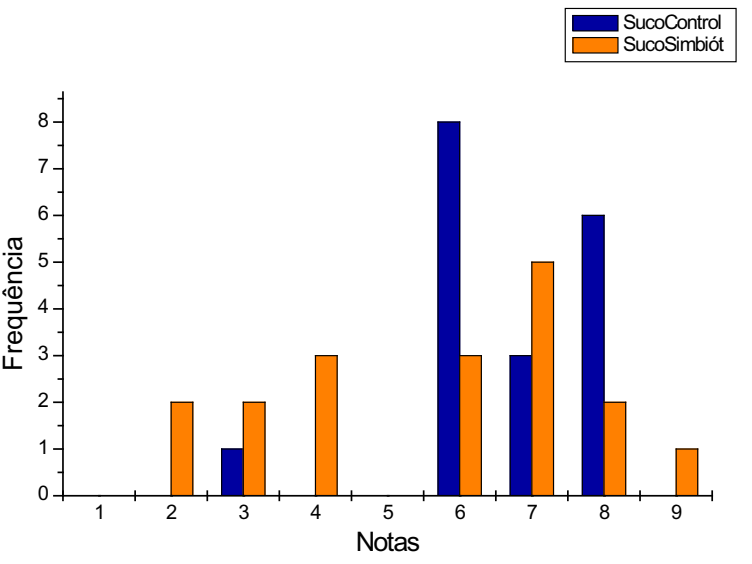

Figura 4. Freqüência das notas atribuídas aos sucos controle e simbiótico através daAnálise Sensorial por Teste Afetivo (18 provadores). Foi utilizada escala hedônica de 9 pontos: 1 - desgostei mutíssimo; 9 - gostei muitíssimo. Nota $<5=$ produto rejeitado, nota $5=$ indiferença, nota $>5=$ produto aceito. Não houve diferença estatística nas notas atribuídas ao suco simbiótico e ao comercial. 
Para o desenvolvimento de um suco simbiótico é importante que este possua o nível de probióticos recomendado pela legislação, e também apresente boas características sensoriais. Estas características desempenham um papel importante na aceitação dos consumidores. Assim, para trabalhos futuros são indicados novos testes para verificar qual seria o período em que os microrganismos permaneceriam viáveis na formulação desenvolvida.

\section{A AGÊNCIA NACIONAL DE VIGILÂNCIA} SANITÁRIA (ANVISA, 2006) considera Lactobacillus acidophilus um microrganismo probiótico. Para comercialização de um produto com alegação de probiótico, no rótulo do mesmo deve constar a seguinte frase: "(indicar a espécie do microrganismo) (probiótico) contribui para o equilibrio da microbiota intestinal. Seu consumo deve estar associado a uma dieta equilibrada e hábitos de vida saudáveis". Além disso, deve-se também declarar, próximo à alegação, a quantidade do probiótico em unidades formadoras de colônias (UFC) contidas na porção diária do produto pronto para consumo. A empresa fabricante deve apresentar laudo de análise do produto para comprovar a quantidade do microrganismo viável até o final do prazo de validade do produto.

\section{Conclusão}

O suco de uva simbiótico desenvolvido pela adição de $10^{8} \mathrm{UFC} / \mathrm{mL}$ de Lactobacillus acidophilus e $10 \%$ de oligofrutose, incubado a $8{ }^{\circ} \mathrm{C}$ por 8 dias, apresentou a contagem mínima necessária para um produto ser considerado probiótico.

Os provadores, através do teste triangular, diferenciaram o suco controle do suco simbiótico. Entretanto, no teste afetivo não houve diferença entre as notas atribuídas pelos provadores ao suco controle e ao simbiótico.

\section{Referências}

AGÊNCIANACIONAL DE VIGILÂNCIA SANITÁRIA - ANVISA. Alimentos com alegações de propriedades funcionais e ou de saúde, novos alimentos/ingredientes, substâncias bioativas e probióticos. Disponível em: $<$ www.anvisa.gov.br>. Acesso em: 20 maio 2006.

BRASIL. Ministério da Agricultura Pecuária e Abastecimento. Resolução $\mathrm{n}^{\circ} 05$, de 13 de novembro de 2000. Padrão de identidade e qualidade de leites fermentados. Diário Oficial [da] República Federativa do Brasil, Brasília, p. 2000.

CHARTERIS, W. P.; KELLY, P. M.; MORELLI, L.; COLLINS, J. K. Ingredient selection criteria for probiotic microorganisms in functional dairy foods. International Journal of Dairy Technology, Huntingdon, v. 51, n. 4, p. 123-136, 1998.

FULLER, R. A review: probiotics in man and animals. Journal of Applied Bacteriology, Oxford, v. 66, n. 5, p. 365-378, 1989.

GERMAN, B.; SCHIFFRIN, E. J.; RENIERO, R.; MOLLET, B.; PFEIFER, A.; NEESER, J. R. The development of functional foods: lessons from the gut. Trends in Biotechnology, Amsterdam, v. 17, n. 12, p. 492-499, 1999.

GIBSON, G. R.; ROBERFROID, M. Dietary modulation of the human colonic microbiota: introducing the concept of prebiotics. Journal of Nutrition, Philadelphia, v. 125, n. 6, p. 1401-1412, 1995.

GOMES, A. M. P., MALCATA, F. X. Agentes probióticos em alimentos: aspectos fisiológicos e terapêuticos, e aplicações tecnológicas. Boletim de Biotecnologia de Alimentos, São Paulo, n. 64, p. 12-22, 1999.

JAIN, P. K.; MCNAUGHT, C. E.; ANDERSON, A. D. G.; MACFIE, J.; MITCHELL, C. J. Influence of synbiotic containing Lactobacillus acidophilus La-5, Bifidobacterium lactis Bb 12, Streptococcus thermophilus, Lactobacillus bulgaricus and oligofructose on gut barrier function and sepsis in critically ill patients: a randomised controlled trial. Clinical Nutrition, Kidlinton, v. 23, n. 4, p. 467-475, 2004.

MARTÍNEZ-VILLALUENGA, C.; FRÍAS, J.; GÓMEZ, R.; VIDAL-VALVERDE, C. Influence of addition of raffinose family oligosaccharides on probiotic survival in fermented milk during refrigerated storage. International Dairy Journal, Barking, v. 16, n. 7, p. 768-774, 2006.

RAO, V. A. The prebiotic properties of oligofructose at low intake levels. Nutrition Research, Tarrytown, v. 21, n. 6, p. 843-848, 2001. 